\title{
Rapid High Resolution Visible Light 3D Printing
}

\author{
Dowon Ahn, Lynn M. Stevens, Kevin Zhou, and Zachariah A. Page* \\ Department of Chemistry, The University of Texas at Austin, 105 East $24^{\text {th }}$ Street, Stop A5300, Austin, \\ Texas 78712, United States. \\ *email: zpage@utexas.edu
}

\begin{abstract}
Light-driven 3D printing to convert liquid resins into solid objects (i.e., photocuring) has traditionally been dominated by engineering disciplines, yielding the fastest build speeds and highest resolution of any additive manufacturing process. However, the reliance on high energy UV/violet light derived from decades of photolithography research, limits the materials scope due to degradation and attenuation (e.g., absorption and/or scattering). Chemical innovation to shift the spectrum into more mild and tunable visible wavelengths promises to improve compatibility and expand the repertoire of accessible objects, including those containing biological compounds and multi-material structures. Photochemistry at these longer wavelengths currently suffers from slow reaction times precluding its utility. Herein, novel panchromatic photopolymer resins were developed and applied for the first time to realize rapid high resolution visible light 3D printing. The combination of electron deficient iodonium and rich borate co-initiators were critical to overcoming the speed-limited photocuring with visible light. Furthermore, azo-dyes were identified as vital resin components to confine curing to irradiation zones, improving spatial resolution. A unique screening method was used to streamline optimization (e.g., exposure time and azo-dye loading) and correlate resin composition to resolution, cure rate, and mechanical performance. Ultimately, a versatile and general visible light-based printing method was shown to afford 1) stiff and soft objects with feature sizes < $100 \mu \mathrm{m}$, 2) build speeds up to $45 \mathrm{~mm} / \mathrm{h}$, and 3) mechanical isotropy, rivaling modern UV-based 3D printing technology and providing a foundation from which bio- and composite-printing can emerge.
\end{abstract}




\section{Introduction}

3D printing has revolutionized the way the world creates, influencing nearly every aspect of modern society - from the consumer market to aerospace and medical technologies. ${ }^{1-4}$ With the help of computer-aided design, digital objects with customized form factors are realized through successive layering of material (i.e., additive manufacturing). The technology has gained traction in-part by overcoming efficiency issues and intermediary processes, such as milling, carving, and machining, affiliated with conventional manufacturing. To date, engineering feats have dominated the arena of $3 \mathrm{D}$ printing, providing a number of elegant methods via extrusion, powder bed fusion, jetting, and light induced polymerization (e.g., stereolithography, SLA, and digital light processing, DLP). ${ }^{1-4}$ Among them, SLA and DLP utilize light to transform matter from liquid resins to solid objects (i.e., photocuring). ${ }^{5-12}$ DLP in particular has drawn wide attention owing to several attractive features, namely some of the fastest build rates (> $100 \mathrm{~mm} / \mathrm{h}$ or $<5$ s/layer), highest feature resolution ( $<100 \mu \mathrm{m}$ features), wide ink viscosity tolerance (up to $\sim 5,000$ $\mathrm{CP}$ ), small footprint (fits on a standard benchtop), and low cost (starting at $\sim \$ 300)$. $^{2,5,7}$ However, the demand for faster printing, milder operating conditions, better resolution, and a wider materials scope continues to drive research efforts and provides an opportunity for chemical innovation.

Contemporary photocuring processes in 3D printing are initiated by high energy ultraviolet (UV) light, which provides rapid polymerization and correspondingly short build times ( seconds). As an alternative, visible light offers numerous benefits, including reduced cost and energy of irradiation from readily available and modular light emitting diodes (LEDs), improved biocompatibility and functional group tolerance, greater depth of penetration, and reduced scattering (Figure 1). ${ }^{10,12-16}$ As such, visible light photocuring has the potential to enable next generation designer material fabrication, including, hydrogels containing live cells, ${ }^{17}$ opaque composites, ${ }^{16}$ and wavelength-selective multi-material structures ${ }^{18-23}$ that promise to advance a range of applications, from structural plastics to tissue engineering and soft robotics.

Nonetheless, the utility of low energy visible LEDs (>420 nm), to the best of our knowledge, has never been demonstrated for SLA- or DLP-based 3D printing. In-part this is due to a lack of commercially available 3D printers with monochromatic visible LEDs, but the grand challenge is to achieve efficient photocuring at these wavelengths to facilitate rapid builds ( $>10 \mathrm{~mm} / \mathrm{h}$ or $<50 \mathrm{~s} /$ layer) with high resolution (< 
$100 \mu \mathrm{m}$ features) and compete with contemporary UV/violet light based additive manufacturing. To improve visible light photocuring efficiency requires a chemical understanding of how reactive curing agents (e.g., radicals or ions) are generated.

Following absorption of light by a chromophore, photocuring can occur by one of two mechanisms: (i) direct photolysis of a photoinitiator (PI) or (ii) electron/energy transfer from a photosensitizer (PS) to a co-initiator followed by bond scission to generate radicals or ions. ${ }^{24-28}$ Although PI compounds often provide rapid photocuring, they rely on a "forbidden" $n \rightarrow \pi^{*}$ transition, which generally corresponds to weak absorption that is restricted to short wavelengths of light $(<420 \mathrm{~nm}$, violet $)$ - exceptions being titanocenes $^{10}$ and acylgermanes ${ }^{29}$ that absorb up to $\sim 500 \mathrm{~nm}$ (blue/green). In contrast, the use of a PS enables excitation via a $\Pi \rightarrow \pi^{*}$ transition, providing strong absorption that can extend to longer wavelengths (> $500 \mathrm{~nm}$, green to NIR). Leaders in materials photochemistry have demonstrated visible-NIR photocuring of a variety of resins, primarily comprising acrylic and epoxy monomers and crosslinkers. ${ }^{10,30,31}$ However, due to the multi-step reaction mechanism (e.g., energy/electron transfer) the cure times are typically slow (> $60 \mathrm{~s}$ ) and require relatively high irradiation intensities $\left(>20 \mathrm{~mW} / \mathrm{cm}^{2}\right)$, making them impractical for standard DLP technology.

Herein, rapid visible light driven chemistry using a novel three component system is developed, characterized, and systematically implemented in high resolution 3D printing. Resin optimization was facilitated by real time Fourier transform infrared (RT-FTIR) spectroscopy and photorheology, and printing was

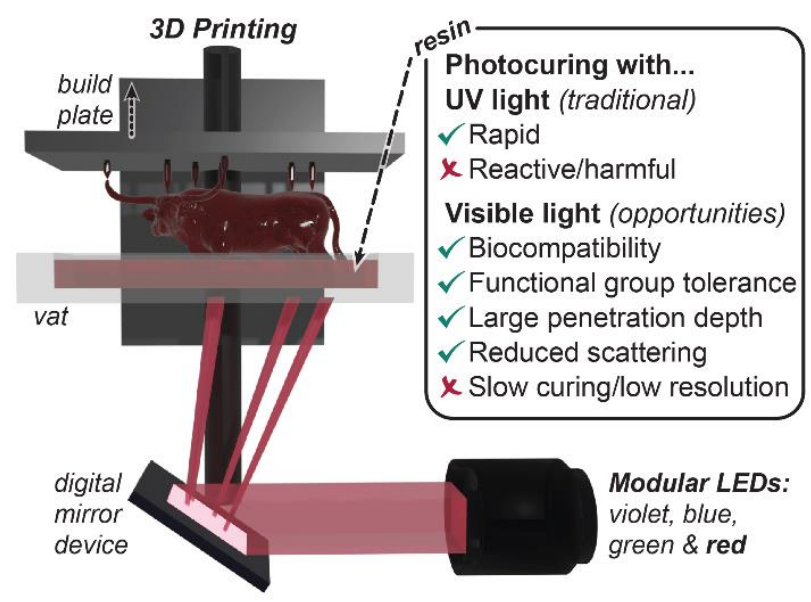

Figure 1. Presented method and opportunities offered by rapid visible light photocuring for 3D printing. Illustration shows the general mechanism for digital light processing (DLP), with exchangeable light emitting diodes (LEDs). 
enabled by a DLP system housing visible LEDs (Figure 1). Comprehensive studies on photocuring rate, feature resolution, and mechanical properties were conducted to inform future development and utility of the present chemistry in materials synthesis for 3D printing and other emerging areas.

\section{Results and Discussion}

To replicate traditional UV-based rapid high resolution DLP 3D printing with low energy visible LEDs necessitated the development of reactive panchromatic resins. These light sensitive polymer resins incorporated monomer, crosslinker, and a PI or PS + co-initiators (i.e., donor, D, and/or acceptor, A). The monomer, crosslinker, and PI were held constant, while various PS and co-initiator compounds were rationally combined to obtain a mixture that enables photocuring on the order of seconds. Specifically, three component systems (PS + two co-initiators) were examined to both promote PS regeneration and potentially double the concentration of radicals produced per photon absorbed (Figure 2a).

Resins comprised dimethyl acrylamide and trimethylolpropane triacrylate as monomer and crosslinker in a 4:1 ratio, respectively. This resin mixture was selected for its good solubilizing characteristics for PS and co-initiator compounds in addition to fast photocuring rate, as demonstrated with a potent PI, bisacylphosphine oxide (BAPO), used here as a violet light $(405 \mathrm{~nm})$ control. For reference, BAPO comprised $0.12 \mathrm{~mol} \%$ (= $0.5 \mathrm{wt} \%$ rel. to monomer+crosslinker) of the resin (contents provided as mol\% for quantitative analysis and wt\% for direct comparison with prior formulation literature $)^{11}$.

Rapid photocuring with blue $(\sim 460 \mathrm{~nm})$, green $(\sim 525 \mathrm{~nm})$, or red $(\sim 615 \mathrm{~nm})$ light was accomplished by replacing the violet absorbing PI with a visible absorbing PS and a donor (D) and acceptor (A) co-initiator. In the present study all resins contain the same electron deficient radical co-initiator $(\mathbf{A})$, [4-(octyloxy)phenyl](phenyl)iodonium hexafluoroantimonate diphenyliodonium $(0.3 \mathrm{~mol} \%=2 \mathrm{wt} \%)$. To find the optimal partner for A, different electron rich co-initiators (D) (e.g., amine, silane, and organoborate derivatives) were screened together with various PSs (e.g., camphorquinone, xanthene, cyanine, and porphyrin derivatives). Initial qualitative assessments of visible light curing (e.g., blue, green, or red) were accomplished by irradiating $100 \mu \mathrm{m}$ thick, argon degassed resins between glass microscope slides using a low intensity light $\left(\sim 5 \mathrm{~mW} / \mathrm{cm}^{2}\right)$ until film formation was noted; for comparison this light source is $\sim 2-20 \times$ less 
intense than an unfocused commercial laser pointer $\left(\approx 10-100 \mathrm{~mW} / \mathrm{cm}^{2}\right)$. Subsequently, RT-FTIR spectroscopy was used to more closely analyze a subset of samples that showed solidification in $\lesssim 60 \mathrm{~s}$ (Table S5 in the SI). The organoborate derivative, 2-(butyryloxy)- $N, N, N$-trimethylethan-1-aminium butyltriphenylborate ${ }^{32,33}$, consistently resulted in the fastest curing rates and was thus used in all subsequent resin formulations $(0.04$ mol\% $=0.2$ wt\%) (Figure $2 b)$. In combination with PS compounds (optimized concentration), $\mathrm{H}-\mathrm{Nu} 470(0.02 \mathrm{~mol} \%=0.1 \mathrm{wt} \%)$, Rose Bengal $(0.01 \mathrm{~mol} \%=0.1 \mathrm{wt} \%)$, and zinc tetraphenylporphyrin (ZnTPP, $0.05 \mathrm{~mol} \%=0.3 \mathrm{wt} \%)$, solidification in < $10 \mathrm{~s}$ was demonstrated upon exposure to blue, green, and red light irradiation, respectively. For comparison, curing in $<10$ s was observed for the violet resin, but with $2-10 \times$ the concentration of $\mathrm{PI}$ (BAPO, $0.12 \mathrm{~mol} \%=0.5 \mathrm{wt} \%$ ) relative to each PS (Figure 2a inset images). These components serve as the "active" ingredients in all subsequent resins for photocuring.

An additional component often present in lithographic resins is an opaquing agent (OA), which serves as a "passive" absorber (i.e., does not elicit a chemical reaction) to control the optical path length of incident light and, in-turn, improve resolution and homogeneity of curing (particularly in the z-dimension). ${ }^{34}$ Ideally OAs (e.g., dyes and pigments) operate by absorbing light in the same wavelength range as the PI or PS within the emission profile of the incident light source. Rapid excited state relaxation is desirable for OAs to preclude electron/energy transfer. Effective OAs in 3D printing reduce the penetration depth of light to mitigate cure through (i.e., curing unwanted regions within previous layers of an object). A series of azo-dyes were selected as OAs given that cis/trans isomerization and intramolecular proton transfer (when phenol functionality is present ortho to the azo group) provide the desired rapid relaxation. ${ }^{35}$ Specifically, Sudan I, IV, and black (Figure 2c) were chosen for their good absorption overlap with both PI/PS absorption and LED emission profiles (Figure 2d).

To correlate the effect of optical attenuation on photocuring parameters, the number of photons absorbed by PI and PS relative to the corresponding OA was determined. This was accomplished by measuring the extinction coefficients for each component using UV-vis absorption spectroscopy (Figures S5S11 in the SI) and integrating that with respect to the emission profile for the different LEDs at a particular intensity (see section 'calculating photons absorbed' in the SI for details). Quantitative LED absorption 
by PI and PS compounds at their optimized concentrations (vide supra) was calculated and compared to

(a)
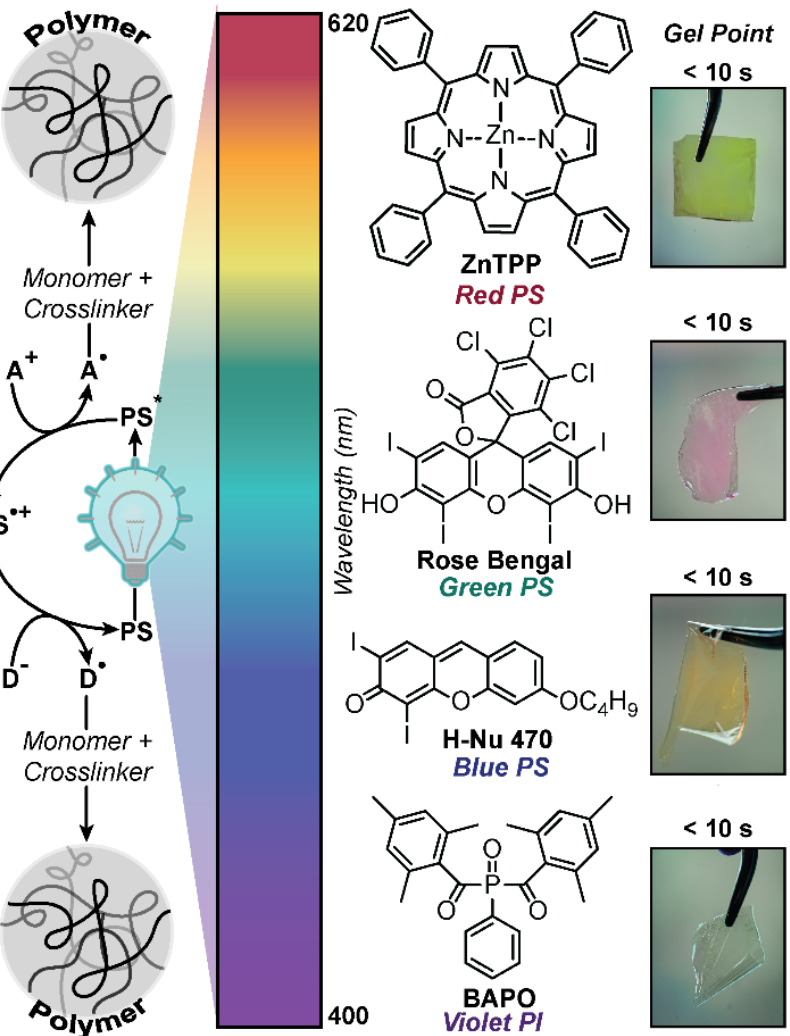

(b)
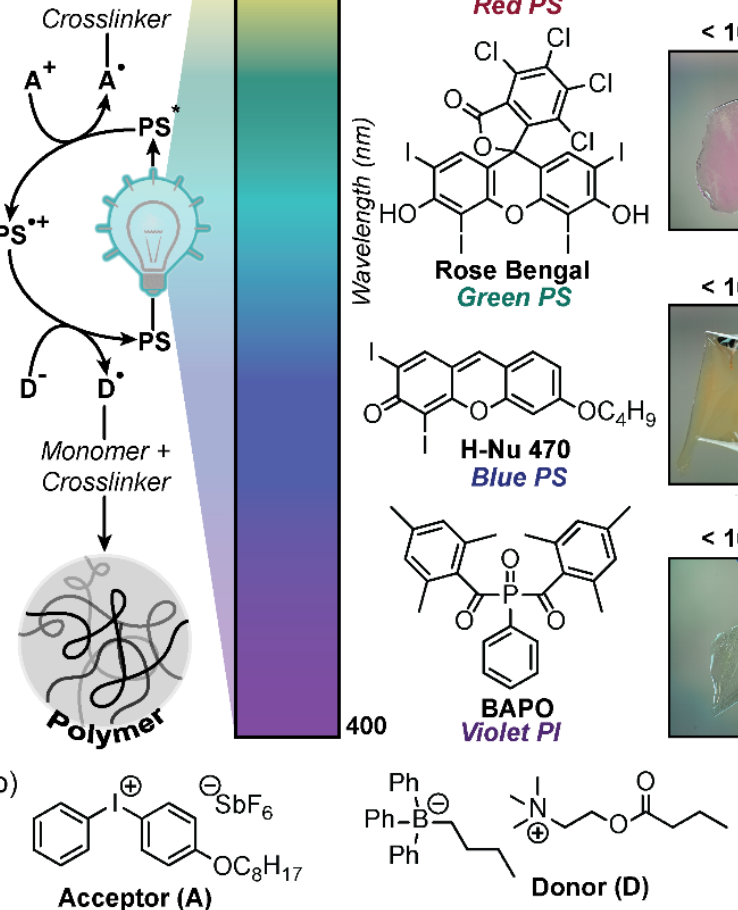

(c)
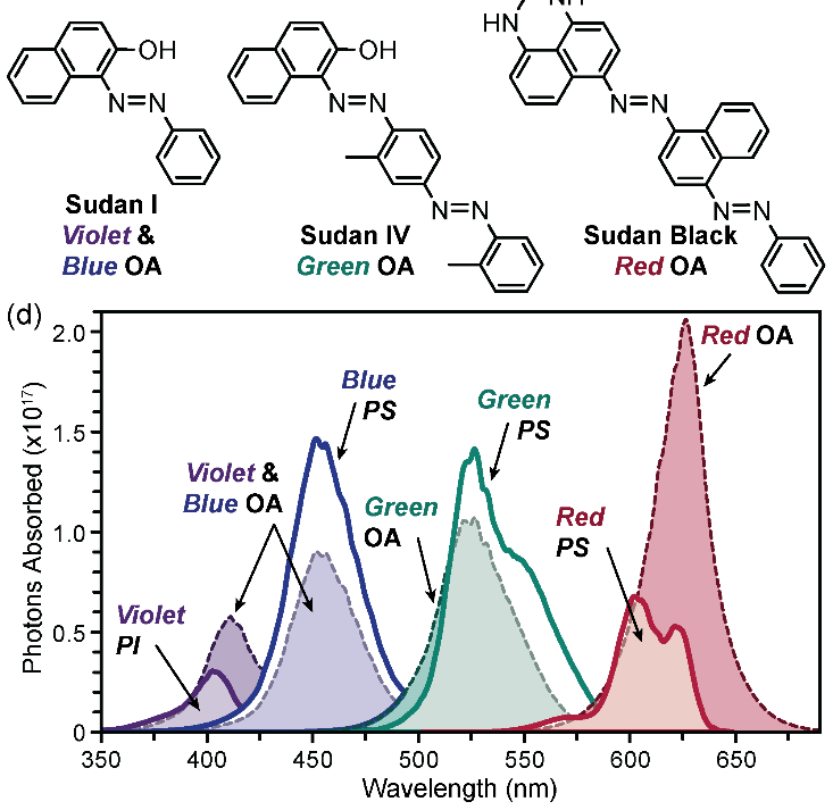

Figure 2. Visible light curing. (a) General mechanism (oxidative quenching) for a three component system (left). Chemical structures of photoinitiator (PI) and photosensitizers (PS), and corresponding pictures of photocured films with qualitative gel times (right). (b) Chemical structures of iodonium acceptor (A) and borate donor (D) co-initiators. (c) Chemical structures of opaquing agents (OAs). (d) Photons absorbed vs wavelength for PI and PS compounds at optimal photocuring concentration and $0.5 \mathrm{mM}$ (red) and $1 \mathrm{mM}$ (rest) OA. Light exposure is from calibrated violet (405 nm), blue $(460 \mathrm{~nm})$, green (525 nm), and red $(615 \mathrm{~nm})$ LEDs at the DLP 3D printer image plane. 
that for OAs at $0.5 \mathrm{mM}$ (Sudan Black) and $1 \mathrm{mM}$ (rest) (for reference $1 \mathrm{mM}=0.01 \mathrm{~mol} \%$ in the prescribed resin). This analysis revealed the following percent absorption of each LED: (violet) $8 \%$ PI and 19\% OA, (blue) 53\% PS and 33\% OA, (green) 57\% PS and 43\% OA, and (red) 17\% PS and 50\% OA (see Table S3 in the SI). Overall, the three OAs and the blue and green PS compounds absorbed significantly more than the violet PI and red PS. The red light absorbing OA was particularly potent, as can be seen in Figure $\mathbf{2 d}$ where it is the tallest peak at half the concentration relative to the other OAs. Another important note is that the green light absorbing OA had reduced overlap with the corresponding PS at longer LED wavelengths $(\sim 570+\mathrm{nm})$ (Figure 2d), which may result in unwanted cure through. Based on these values, we hypothesized that the resin activated by blue light would need the least amount of OA to prevent cure through and that the red light sensitive resin would be greatly influenced by small fluctuations in [OA]. Notably, while the red PS (ZnTPP) absorbed a fraction of the photons $(\sim 3 \times$ fewer) relative to the other PS compounds at different wavelengths, it maintained rapid photocuring $(<10 \mathrm{~s})$ at low light intensity $\left(\sim 5 \mathrm{~mW} / \mathrm{cm}^{2}\right)$, making it an excellent candidate for 3D printing.

To demonstrate the utility of these novel resins in 3D printing, a custom DLP (Figure S1 in the SI) with modular visible LEDs (peak emission $\approx 405,460,525,615 \mathrm{~nm}$ ) was constructed. The minimum volume element (voxel) on the printer has lateral dimensions of $20 \times 20 \mu \mathrm{m}^{2}$ and a vertical dimension of $25 \mu \mathrm{m}$ (i.e., layer thickness). To examine resolution and mechanical properties of prints in conjunction with curing rates necessitated the use of a $100 \mu \mathrm{m}$ layer thickness, since RT-FTIR and photorheology monitoring required sample thickness $\geq 100 \mu \mathrm{m}$ for adequate signal. Beneficially, the thicker layers facilitate faster builds (vide infra). A novel printing method, referred to here as "resolution print", was developed to efficiently optimize resolution and build speed. Critically, the method provides control over exposure time within different locations of a single layer, which facilitated rapid screening (Figure 3a). This printing method was used to correlate exposure time/layer and OA concentration, $[\mathrm{OA}]$, to lateral $(\mathrm{x}, \mathrm{y})$ and vertical (z) resolution. In this assessment, each resolution print contained a set of squares that were simultaneously printed, varying exposure time/layer. In the bottom half of each square was an array of smaller patterns that were 1 to 16 pixels wide, used to characterize resolution. As shown in Figure $\mathbf{3 b}$, a 12 square array was printed $\left(4 \times 4 \mathrm{~mm}^{2}\right.$ each square) with $4 \times 100 \mu \mathrm{m}$ layers, and exposure times from 1 to 
12 s/layer (time in seconds engraved in each square). For ease of handling, the square array was printed on a rectangular base (12 layers at 12 s/layer). Thus, a single print contained resolution information about 12 exposure times at a particular [OA], streamlining optimization.

Resolution prints were accomplished using violet, blue, green, and red resins with varying amount of the corresponding OA to identify optimal resin composition and exposure time/layer (Figure 2c). As the [OA] was increased the first square pattern to appear occurred at longer exposure times, indicating an inverse relationship between build speed and [OA]. This suggests that the OA is effectively competing for photons with that of the PI or PS present (i.e., passive absorption). Even with OA present, patterns were observed within $2 \mathrm{~s}$ for all resin formulations, demonstrating the ability to perform rapid visible light curing (Figure 3b). Of note, for green and red light sensitive samples a blanket of inert gas (e.g., nitrogen or argon) during the print was necessary to achieve these speeds, while violet and blue light printing was less affected. Under ambient conditions polymerizations are delayed for varying lengths of time (i.e., inhibition period). The delay is attributed to quenching of triplet excited states by oxygen, supported by longer inhibition periods observed for the red light PS $\left(\mathrm{ZnTPP}^{36}\right)$ known to provide high triplet yields.

Concomitant with reduced cure rates upon increasing $[\mathrm{OA}]$, was an improved z-resolution as a result of diminished cure through. The z-resolution was characterized by measuring the thickness and angle between the top surface and connecting vertical wall (i.e., sidewall angle, SWA) for each time point on a print using 3D imaging (optical profilometry) (Figure 3c). A representative topographical profile for an 11 s/layer square on an optimized red light resolution print (with $O A$ ) is shown in Figure 3a. Heights and SWA values were determined from an average of 10 positions, using a line measurement tool as depicted in Figure3c(i). Ideal values for the resolution prints reflect the imported digital images, which equated to a height of $400 \mu \mathrm{m}$ (4 layers at $100 \mu \mathrm{m} /$ layer) and a SWA of $90^{\circ}$. Without OA the maximum thickness and SWA did not always occur at the same exposure time/layer (Figures S14-18 in the SI). For example, the red resin without OA had a maximum thickness of $384 \mu \mathrm{m}$ at $5 \mathrm{~s} /$ layer and a SWA of $78^{\circ}$ at $4 \mathrm{~s} /$ layer (Figure 3c, bottom). Additionally, at shorter and longer exposure times both thickness and SWA rapidly decrease, providing a narrow processing window, hindering reproducibility. This is exemplified by setting 
a tolerance of $\pm 5 \%$ for thickness $(400 \pm 20 \mu \mathrm{m})$ and $\pm 17 \%$ for SWA $\left(90 \pm 15^{\circ}\right.$ ), which results in a processing window of $\sim 1 \mathrm{~s}$ without OA present (exposure time/layer $\sim 5 \mathrm{~s}$ ). This short processing window was attributed to incomplete curing and cure through prior to- and post-5 s, respectively. Increasing [OA] resulted in a convergence in exposure time for both optimal thickness and SWA, along with a much wider and more well defined processing window $(\geq 6 s)$. Consistent with our hypothesis, blue resins did not require any OA to mitigate cure through, which was attributed to the strong absorption by the blue PS.

(a) Resolution Print Method

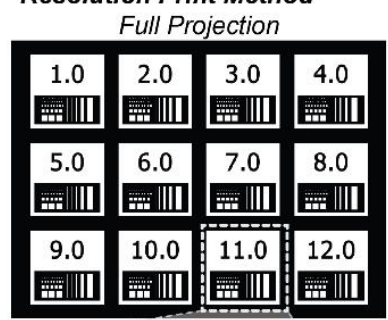

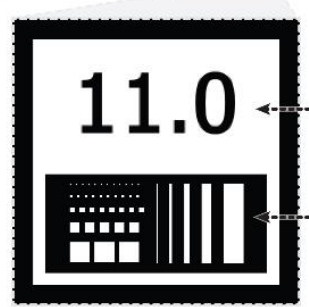

(c)
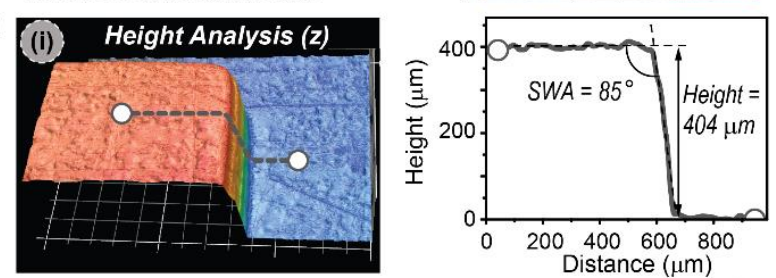
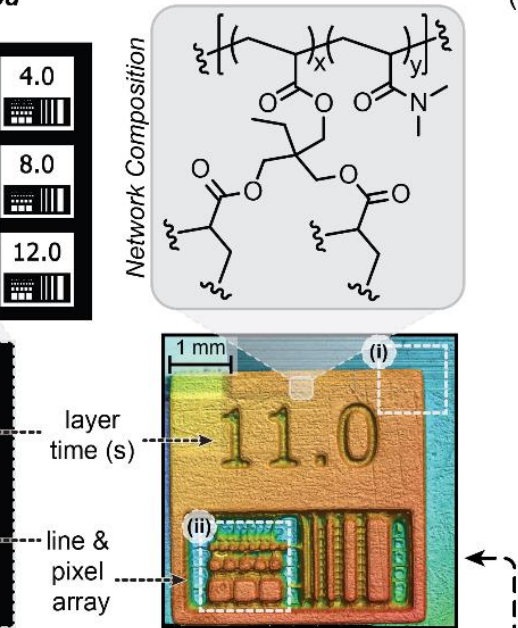

(b) Resolution Prints (by LED color)

\section{Violet}

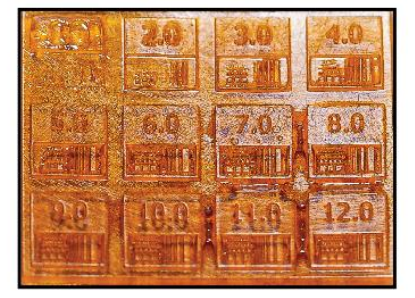

Green

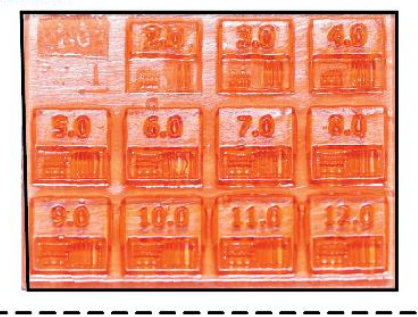

(d) Surface Area Analysis (x,y)

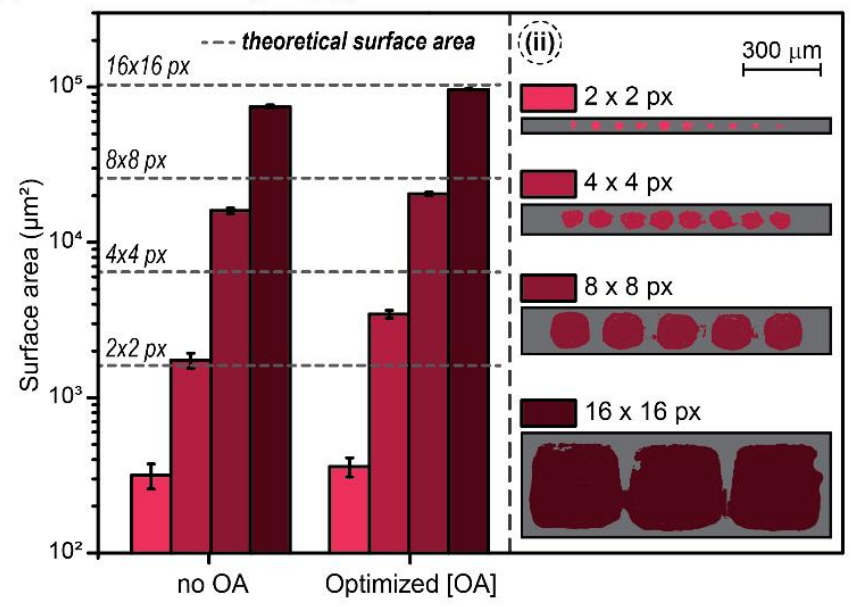

Figure 3. 3D print optimization protocol using the "resolution print" method. (a) Digital projection layer at one second when all squares are illuminated simultaneously (white regions correspond to exposure) (top left). Expanded square for $11 \mathrm{~s}$ exposure/layer showing the line and pixel array for the projection (bottom left) and red light resolution print taken with optical profilometry (bottom right). Chemical composition for photocured stiff resin comprising dimethyl acrylamide and trimethylolpropane triacrylate (top right). (b) Optical images of prints from the four resins by exposure color (100 $\mu \mathrm{m}$ layers, 4 layers for each numbered square on top of 12 base layers). (c) z-resolution analysis: 3D image of a corner of the $11 \mathrm{~s} \mathrm{square}$ outlined as box (i) in the profilometry image above. Height and sidewall angle (SWA) were determined using an average of 10 line traces per corner (with one shown for reference). Graph of thickness and SWA vs exposure time for the red resin printed with and without OA (bottom). Shows how OA improves z-resolution and increases the processing window (theoretical height $=400 \mu \mathrm{m}$ ). (d) $x, y$-resolution analysis: Plot of surface areas measured for $16,8,4$, and 2 pixel wide squares using optical profilometry. Dashed lines represent theoretical surface areas, and error bars represent \pm 1 standard deviation, showing how OA enhances print fidelity and reproducibility of features below $100 \mu \mathrm{m}$ in length. 
Also in-line with our prior observations was the small amount of OA necessary to mitigate cure through for prints with the green resin $(0.003 \mathrm{~mol} \% \mathrm{OA})$ and, to a greater effect, the red resin $(0.0005 \mathrm{~mol} \% \mathrm{OA})$ (Table S1 in the SI). At the optimal [OA] for the red resin an average thickness of $405 \mu \mathrm{m}$ and SWA of $85^{\circ}$ was observed across a wide exposure window from 7-12 s/layer (Figure 3c). Complete thickness, SWA, and processing window data for all resins are provided in the supporting information (Figures S1418). Optimal exposure times/layer fell between 8 and $11 \mathrm{~s}$ for all resins, which corresponds to a build rate of $45-33 \mathrm{~mm} / \mathrm{h}$ (not including the recoating process). Visible light printing was shown to offer wide processing windows to facilitate reproducibility, while providing objects with z-resolution and build speeds that rival contemporary UV-based DLP 3D printers².

To assess $\mathrm{x}, \mathrm{y}$-resolution, surface areas for the pixel arrays were characterized from the topographical images, as shown in Figure 3d(ii). Specifically, 16, 8, 4, and 2 pixel wide pixel arrays were analyzed by imaging the surfaces for both red (Figure 3d) and violet (Figure S19 in the SI) resolution prints. Samples with and without OA were characterized and compared at optimal exposure times (8 and 4 s/layer for violet with and without $O A$, and 11 and 5 s/layer for red with and without OA, respectively). Surface area analysis revealed that the presence of OA results in a better match to theoretical, corresponding with larger SWAs (dashed lines in Figure 3d). The improved x,y-resolution is hypothesized to result from attenuating light outside of predefined irradiation zones (e.g., scattered light). Additionally, it was noted that deviation of surface area from theoretical values increased as feature size decreased. For red prints with optimized $[\mathrm{OA}]$ at an $11 \mathrm{~s}$ exposure time/layer the surface area for decreasing features $(320 \times 320$ to $40 \times 40 \mu \mathrm{m}^{2}$ ) deviated from theoretical values by $6 \%, 20 \%, 46 \%$, and $78 \%$, respectively. The diminished fidelity for smaller features is attributed to incomplete curing. As these polymerizations are exothermic, small features do not generate as much heat as larger features, which can autoaccelerate curing. Irrespective of the feature size, reproducibility was excellent, as evidenced by the small standard deviations in surface area values between same size squares. The present study clearly illustrates that high resolution features $(<100 \mu \mathrm{m})$ can be accomplished reproducibly using visible light 3D printing.

The photopolymerization rates and times to gelation were determined to inform future resin develop- 
ment for low energy visible light 3D printing, among other photocuring applications in imaging, lithography, coatings, and adhesives. ${ }^{9}$ To this end, RT-FTIR spectroscopy and photorheology were accom plished, carefully matching 3D printing conditions (e.g., thickness, atmosphere, and light intensity) (Figure 4a). Specifically, the samples were $100 \mu \mathrm{m}$ thick, under an inert environment (e.g., degassed with $\mathrm{N}_{2}$ or argon), and LED exposure intensities matched those of the 3D printer at the resin vat: $3.3,3.4,1.8$, and $2.1 \mathrm{~mW} / \mathrm{cm}^{2}$ for violet, blue, green, and red, respectively. As a control, data was collected for $10 \mathrm{~s}$ in the dark, showing no polymerization prior to light exposure, which demonstrates the efficient temporal nature of these photosystems. The near IR (NIR) absorption region $\left(8,000-4,000 \mathrm{~cm}^{-1}\right)$ was monitored using RT-FTIR to determine monomer/crosslinker conversion by measuring the disappearance of $\mathrm{C}=\mathrm{C}$ vinylic stretches found at $\sim 6,160 \mathrm{~cm}^{-1} .37$ The inherently weak absorption signals in the NIR (e.g., overtones of mid IR signals) required that samples were $\sim 100 \mu \mathrm{m}$ thick, but enabled the utility of disposable glass slides for facile preparation and measurement (note: glass is NIR transparent, but mid IR opaque).

Under the optimized printing conditions each resin rapidly polymerized, reaching a maximum monomer conversion within $20 \mathrm{~s}$ of turning the light on (Figure $\mathbf{4 b}$ ). Notably, the $C=C$ conversion peaks at $\sim 80 \%$, which is likely due to a reduction in molecular motion post-gelation. Moreover, under ambient conditions a distinct induction period was observed after turning the light on, consistent with the slower printing speed noted previously. Specifically, inhibition times of 2, 5, 8, and $83 \mathrm{~s}$ was observed for violet, blue, green, and red respectively (Table $\mathbf{S 6}$ and Figures $\mathbf{S 2 2}$ in the $\mathbf{S I}$ ), confirming the distinct sensitivity of the red PS (ZnTPP) to oxygen. However, a similar maximum polymerization rate is reached once oxygen is consumed. The similar rate is attributed to either efficient regeneration of the PS in the tricomponent photosystem (Figure 2a) and/or a rate limiting step other than electron/energy transfer, such that removing a fraction of PS upon reaction with oxygen does not alter the apparent rate. These findings reinforces that oxygen removal as an effective strategy to increase photocuring rate and accordingly print speeds. In addition to increasing photopolymerization rate by oxygen removal, the effect of light intensity was examined as a complementary route to further improve curing rate and associated build speeds. At the optimized printing conditions the maximum apparent polymerization rates were $1.33 \pm 0.05 \mathrm{M} / \mathrm{s}, 1.16 \pm$ $0.05 \mathrm{M} / \mathrm{s}, 1.09 \pm 0.09 \mathrm{M} / \mathrm{s}$, and $1.9 \pm 0.3 \mathrm{M} / \mathrm{s}$, for violet, blue, green, and red respectively. Increasing the 
light intensity to $10 \mathrm{~mW} / \mathrm{cm}^{2}$ resulted in a $\sim 1.6-2 \times$ polymerization rate enhancement relative to those obtained at the printer intensities (Table S7 and Figures S26-S33 in the SI).

Photorheology was used to determine gel times and corresponding double bond conversion at the gel point to inform future resin development. Gelation was identified as the crossover between storage and loss modulus, which occurred within 2 to 4 seconds of light exposure. This short gel time is consistent with the first appearance of features on the resolution prints (Figure 3b). Specifically, gel points were 1.8 $\pm 0.2 \mathrm{~s}, 1.9 \pm 0.1 \mathrm{~s}, 3.4 \pm 0.2 \mathrm{~s}$, and $4.2 \pm 0.2 \mathrm{~s}$ for violet, blue, green, and red respectively (Figures S34S37 and Table S8 in the SI). The measured double conversions at the gel points fell within a similar range for all resins $(\sim 5-8 \%)$, which is expected given the constant monomer and cross-linker composition in each resin (Figure $\mathbf{4 b}$, semi-transparent grey bar). The $\mathrm{C}=\mathrm{C}$ conversion for each resin at the optimized 3D printing exposure time/layer ( $\sim-11 \mathrm{~s})$ occurred at $\sim 50-70 \%$, which falls below the maximum conver-
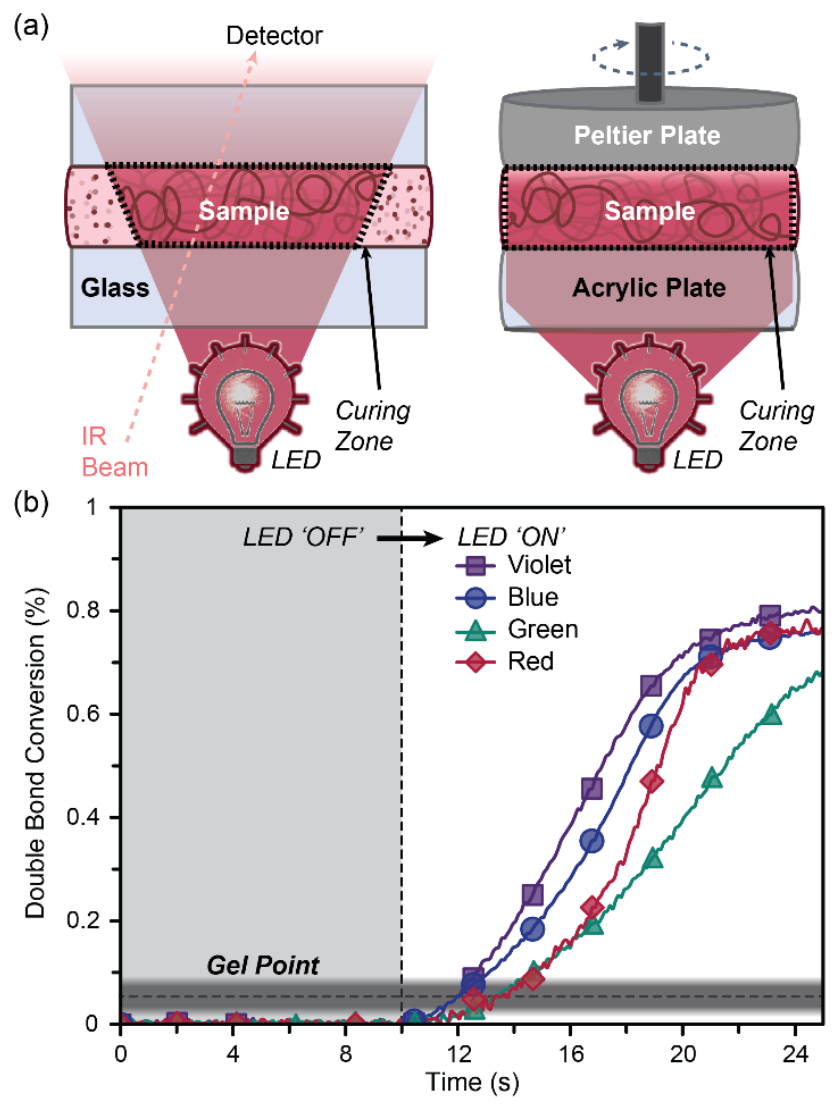

Figure 4. Photopolymerization rate and gel point characterization. (a) Schematic representations of the RT-FTIR and photorheology setups. (b) Plot of double bond conversion vs time for violet, blue, green, and red photopolymerizations. Experiments were performed under an inert atmosphere at an exposure intensity matching the 3D printer. Light exposure began at $10 \mathrm{~s}$, highlighting the rapid temporal response. The gradient bar represents the gel point range found for all four samples $(\sim 5-8 \%$ conversion), which was determined using a combination of photorheology and RT-FTIR.

sion ( $80 \%)$. Therefore, polymerizable vinylic residues should be present at the interface of each layer 
during the 3D printing process, which can facilitate covalent bonding and increase mechanical strength.

To push the boundaries of additive manufacturing from traditional hobbyist printing and models to applications that require both precision and structural integrity necessitated a thorough understanding of mechanical performance. To this end, dogbones (ASTM D638) from each resin formulation were 3D printed under optimized conditions and subjected to tensile testing (Figure 5). Mechanical properties of interest included stiffness ( $\boldsymbol{E}$, Young's modulus), yield strength $\left(\boldsymbol{\sigma}_{\boldsymbol{y}}\right)$, and strain at fracture $\left(\boldsymbol{\varepsilon}_{\boldsymbol{f}}\right)$. Following standard protocols, all objects were post-cured with UV light prior to tensile testing (20 min., centered at $\sim 370 \mathrm{~nm}$ ). As a control, post-curing red light printed dogbones with red light in place of UV light provided nearly identical mechanical performance (Figure $\mathbf{S} 38$ in the SI). Excitingly, $\boldsymbol{E}, \boldsymbol{\sigma}_{\boldsymbol{y}}, \boldsymbol{\varepsilon}_{\boldsymbol{f}}$ were consistent for all resins, suggesting that the presence of a tricomponent photoactive system does not alter mechanical performance: $\boldsymbol{E}=997 \pm 62 \mathrm{MPa}$ (violet), $1022 \pm 63 \mathrm{MPa}$ (blue), $986 \pm 102 \mathrm{MPa}$ (green), and $1043 \pm 94$ MPa (red) (Figure 5a and Table S9).

Mechanical uniformity of visible light printed objects was examined, as layered production is often challenged with an undesirable anisotropic mechanical response with weakness at the boundaries between adjacent layers. ${ }^{39-41}$ To this end, dogbones from the optimized red resin were printed at three different edge-on angles - horizontal $\left(0^{\circ}\right)$, vertical $\left(90^{\circ}\right)$, and diagonal $\left(45^{\circ}\right)$ (Figure 5b). Optical profilometry images of the dogbones printed at different angles clearly show the layers and demonstrates excellent thickness accuracy (102 $\pm 1 \mu \mathrm{m} /$ layer, $100 \pm 2 \mu \mathrm{m} /$ layer, $99 \pm 2 \mu \mathrm{m} /$ layer for horizonal, vertical, and diagonal angles, respectively ) (Figure 5b inset). Compellingly, $\boldsymbol{E}$ values were unperturbed by changing print angle (average $\boldsymbol{E}=1083 \pm 47 \mathrm{MPa}$ ), and only small fluctuations in $\boldsymbol{\sigma}_{\boldsymbol{y}}$ and $\boldsymbol{\varepsilon}_{\boldsymbol{f}}$ were observed: $\boldsymbol{\sigma}_{\boldsymbol{y}}=$ $31 \pm 2 \mathrm{MPa}, 31 \pm 2 \mathrm{MPa}$, and $29 \pm 2 \mathrm{MPa}$ for horizontal, diagonal, and vertical angles, respectively (Figure 5b and Table S10 in the SI). These minor differences are in accord with other objects printed via UV-based vat photopolymerization. ${ }^{39-41}$

To demonstrate the versatility of visible light 3D printing, mechanically and chemically disparate objects were prepared (e.g., stiff vs soft and hydrophobic vs hydrophilic). The previous resin provided a stiff and brittle object, which was attributed to the high glass transition temperature for poly(dimethyl acrylamide) $\left(T_{g} \approx 90^{\circ} \mathrm{C}\right)$ in conjugation with a large amount of crosslinker (i.e., 20 wt\% triacrylate rel. to monomer). 
3D printing soft and extensible objects remains an active area of research due to the challenges that arise from their natural tendency to deform during prints. ${ }^{42-44}$ A resin comprising 2-hydroxyethyl acrylate monomer with a correspondingly low polymer $T_{g}\left(\approx-15^{\circ} \mathrm{C}\right)$ and a small amount of crosslinker, tetra(ethylene glycol) diacrylate ( $0.1 \mathrm{wt} \%$ rel. to monomer), was formulated to provide a softer and more extensible
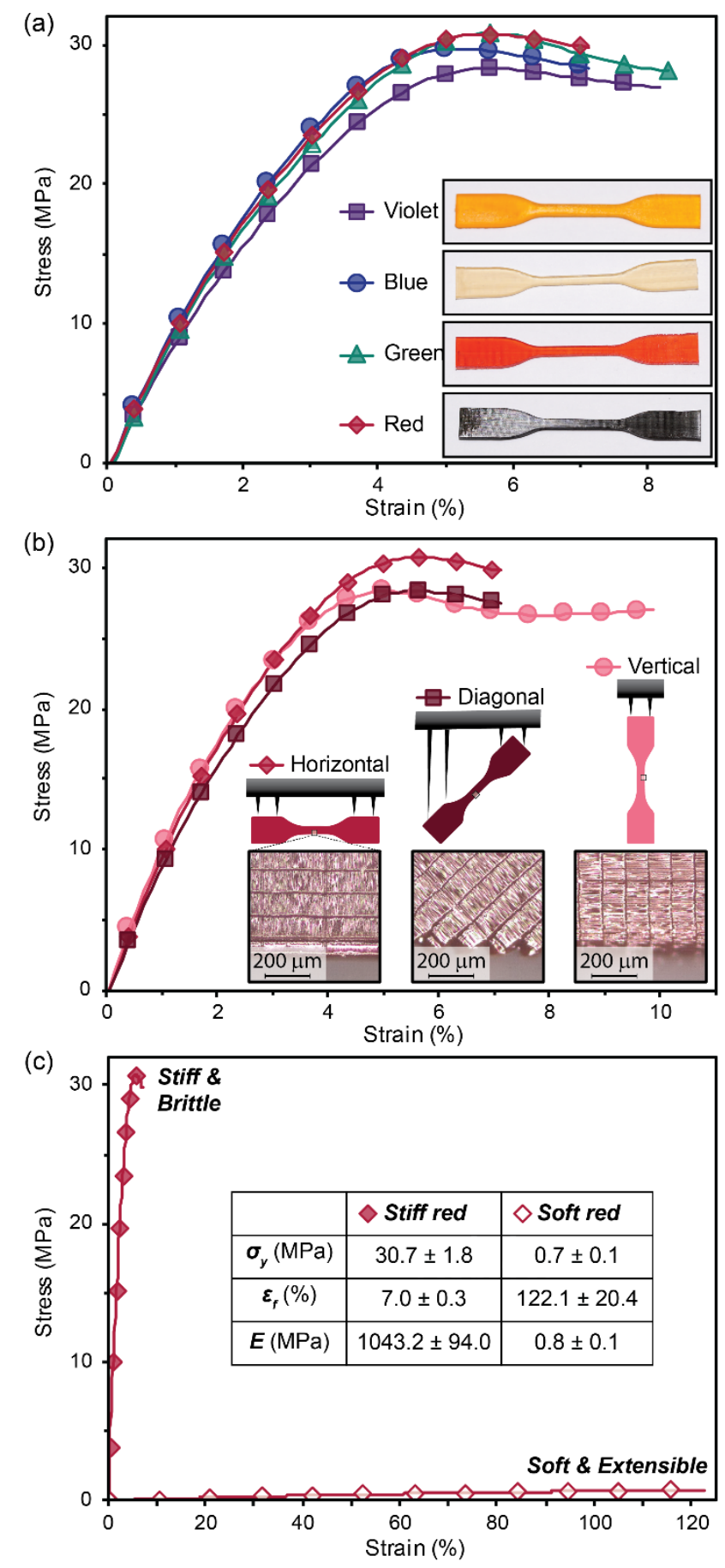

Figure 5. Mechanical testing of 3D printed dogbones (a) Stress-strain curves of violet, blue, green, and red dogbones (b) Red dogbones printed at three different edge-on angles (horizontal, diagonal, and vertical). Insets show a drawing of each dogbone orientation, with supports to the build platform shown, and close up images to visualize each layer angle. (c) Stress-strain curves of stiff and elastic red resin. Inset shows a table highlighting the disparate mechanical properties. 
material. With the optimized red light photosystem, dogbones were 3D printed with the new soft resin formulation. Tensile testing revealed a $E=0.8 \pm 0.1 \mathrm{MPa}$, which is three orders of magnitude lower than those from the stiff resin (Figure 5c). Moreover, swelling tests in water for six cubes printed with either stiff or soft resin qualitatively highlight the difference in chemical makeup (Figure S40). The soft cubes had a much larger water uptake (177 $\pm 1 \%$ increase in weight) compared to the stiff resin $(54 \pm 2 \%$ increase in weight), which is attributed to a combination of lower crosslink density and increased hydrophilicity of the alcohol-laden soft network (Table S13 in the SI). The contrasting mechanical and chemical properties of objects printed with the same visible light photosystem demonstrates the versatility of the present method, which will provide access to a myriad of materials without the need for harmful UV light.

As a final demonstration, an octet truss was printed to show how visible light additive manufacturing can be used to rapidly provide high resolution complex (e.g., hierarchical) 3D objects that are challenging or impossible to produce using traditional manufacturing processes. The octet truss was printed with the original stiff red resin using both $100 \mu \mathrm{m}$ (Figure S41) and $25 \mu \mathrm{m}$ layer thickness (Figure 6). Decreasing the layer thickness facilitated reduced exposure times/layer, from $11 \mathrm{~s}$ at $100 \mu \mathrm{m}$ layers to $8 \mathrm{~s}$ at $25 \mu \mathrm{m}$ layers, correlating to a build rate of $\sim 7 \mathrm{~mm} / \mathrm{h}$. Scanning electron microscopy (SEM) images shows the high printing fidelity along with individual layers at a pitch of $25.1 \pm 0.6 \mu \mathrm{m}$. Interestingly, the final object was also red emissive under a handheld $365 \mathrm{~nm}$ UV light (Figure S42 in the SI), which demonstrates

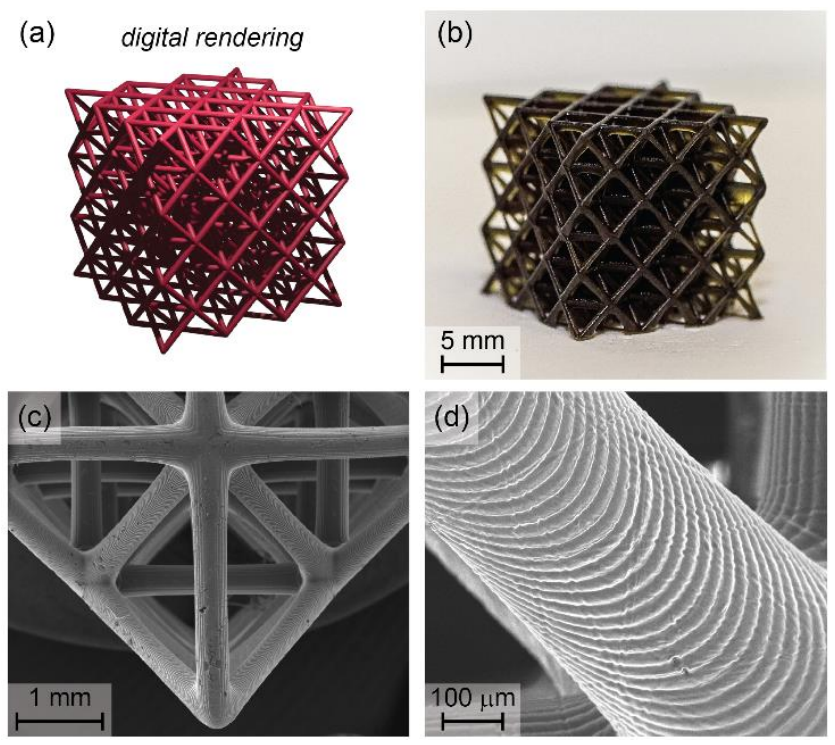

Figure 6. Hierarchical octet truss as a complex 3D print demonstration. (a) Digital rendering. (b) Photograph of the printed object using the stiff red resin composition and red light exposure $(\sim 2.1 \mathrm{~mW} / \mathrm{cm} 2)$ for $8 \mathrm{~s} / 25 \mu \mathrm{m}$ layer. (c,d) Scanning electron microscope images at different magnification showing the structural hierarchy. 
that a certain amount of embedded PS (ZnTPP) remains intact, providing an avenue towards functional 3D prints (i.e., "smart" plastics). Additional 3D prints can be found in the supporting information file (Figure $\mathbf{S 4 3}$ in the SI). These sophisticated structures with custom form factors emphasize the utility of visible light 3D printing to seamlessly reproduce complex digital inputs with excellent feature fidelity.

\section{Concluding Remarks}

Novel photopolymer resins that rapidly react upon irradiation with visible light across the spectrum were described and systematically examined for use in high resolution DLP 3D printing. Key discoveries and developments include rapid photocuring from combined iodonium (donor) and borate (acceptor) co-initiators, resolution enhancements from visible light absorbing azo-dyes (OA), and efficient optimization using a custom "resolution print" method. A DLP 3D printer with exchangeable LEDs provided rapid build speeds from 33 to $45 \mathrm{~mm} / \mathrm{h}$ for low intensity violet, blue, green, and red light exposure $\left(\sim 2-3 \mathrm{~mW} / \mathrm{cm}^{2}\right)$. RT-FTIR and photorheology were used to judiciously correlate chemical composition of resins to photocuring rate, $\mathrm{x}, \mathrm{y}, \mathrm{z}$-resolution, build speed, and mechanical properties. Inclusion of OA compounds into the resin formulation was shown to enhance resolution, with features $<100 \mu \mathrm{m}$ in the lateral dimension and $25 \mu \mathrm{m}$ layer thickness, while simultaneously enhancing reproducibility by widening the processing window prior to cure through from $\sim 1$ to $\geq 6 \mathrm{~s}$. Mechanical properties were shown to be nearly independent of curing wavelength and the print orientation, demonstrating the production of relatively isotropic parts. Versatility was highlighted by rapidly producing both stiff and soft objects with stiffness ranging from $\sim 1480$ to $0.8 \mathrm{MPa}$. Finally, objects with intricate form factors were printed as a testament to the 3D capabilities offered by the present technique. As a future endeavor, increasing light intensity (up to 10 $\left.\mathrm{mW} / \mathrm{cm}^{2}\right)$ provides an avenue towards even faster visible light based 3D printing $(\sim 1.5-2 \times)$. The foundational studies provided herein will serve as a roadmap for further development of visible and NIR photocuring with excellent spatial control, material compatibility, and utility that extends beyond 3D printing (e.g., coatings, adhesives, imaging, and lithography). As a result, we envision numerous exciting and transformative interdisciplinary research opportunities in academic, industrial, and medical sectors, including hierarchical multimaterial fabrication, fiber reinforced composites, and cell-laden hydrogels. 


\section{Conflicts of interest}

There are no conflicts to declare.

\section{Acknowledgements}

We thank the ARO STIR program of the Department of Defense (W911NF1910310) and Robert A. Welch Foundation (F-2007) for financial support. The authors acknowledge the use of shared research facilities supported in part by the Texas Materials Institute, the Center for Dynamics and Control of Materials: an NSF MRSEC (DMR-1720595), and the NSF National Nanotechnology Coordinated Infrastructure (ECCS-1542159). We thank Dr. Yun-Ho Jang at MonoPrinter for the custom DLP 3D printer and software design.

\section{Abbreviations}

SLA, Stereolithography; DLP, Digital light processing; UV, ultraviolet; LED, light emitting diode; PI, photoinitiator; PS, photosensitizer; RT-FTIR, real time Fourier transform infrared; OA, opaquing agent; ASTM, American Society for Testing and Materials

\section{References}

(1) Narupai, B.; Nelson, A. 100th Anniversary of Macromolecular Science Viewpoint: Macromolecular Materials for Additive Manufacturing. ACS Macro Lett. 2020, 627-638.

(2) Wallin, T. J.; Pikul, J.; Shepherd, R. F. 3D Printing of Soft Robotic Systems. Nat. Rev. Mater. 2018, 3 (6), 84-100.

(3) Ligon, S. C.; Liska, R.; Stampfl, J.; Gurr, M.; Mülhaupt, R. Polymers for 3D Printing and Customized Additive Manufacturing. Chem. Rev. 2017, 117 (15), 10212-10290.

(4) Truby, R. L.; Lewis, J. A. Printing Soft Matter in Three Dimensions. Nature 2016, 540 (7633), 371-378.

(5) Tumbleston, J. R.; Shirvanyants, D.; Ermoshkin, N.; Janusziewicz, R.; Johnson, A. R.; Kelly, D.; Chen, K.; Pinschmidt, R.; Rolland, J. P.; Ermoshkin, A.; et al. Continuous Liquid Interface Production of 3D Objects. Science. 2015, 347 (6228), 1349-1352.

(6) Kelly, B. E.; Bhattacharya, I.; Heidari, H.; Shusteff, M.; Spadaccini, C. M.; Taylor, H. K. Volumetric Additive Manufacturing via Tomographic Reconstruction. Science. 2019, 363 (6431), 1075-1079.

(7) Walker, D. A.; Hedrick, J. L.; Mirkin, C. A. Rapid, Large-Volume, Thermally Controlled 3D Printing Using a Mobile Liquid Interface. Science. 2019, 366 (6463), 360-364.

(8) Jung, K.; Corrigan, N.; Ciftci, M.; Xu, J.; Seo, S. E.; Hawker, C. J.; Boyer, C. Designing with Light: Advanced 2D, 3D, and 4D Materials. Adv. Mater. 2019, 1903850.

(9) del Barrio, J.; Sánchez-Somolinos, C. Light to Shape the Future: From Photolithography to 4D Printing. Adv. Opt. Mater. 2019, 7 (16), 1900598. 
(10) Bagheri, A.; Jin, J. Photopolymerization in 3D Printing. ACS Appl. Polym. Mater. 2019, 1 (4), 593-611.

(11) Zhang, J.; Xiao, P. 3D Printing of Photopolymers. Polym. Chem. 2018, 9 (13), 1530-1540.

(12) Chatani, S.; Kloxin, C. J.; Bowman, C. N. The Power of Light in Polymer Science: Photochemical Processes to Manipulate Polymer Formation, Structure, and Properties. Polym. Chem. 2014, 5 (7), 2187-2201.

(13) Xiao, P.; Zhang, J.; Dumur, F.; Tehfe, M. A.; Morlet-Savary, F.; Graff, B.; Gigmes, D.; Fouassier, J. P.; Lalevée, J. Visible Light Sensitive Photoinitiating Systems: Recent Progress in Cationic and Radical Photopolymerization Reactions under Soft Conditions. Prog. Polym. Sci. 2015, 41 (C), 32-66.

(14) Bagheri, A.; Bainbridge, C.; Jin, J. Visible Light-Induced Transformation of Polymer Networks. ACS Appl. Polym. Mater. 2019, 1 (7), 1896-1904.

(15) Dumur, F. Recent Advances on Visible Light Metal-Based Photocatalysts for Polymerization under Low Light Intensity. Catalysts 2019, 9 (9), 736.

(16) Strehmel, B.; Schmitz, C.; Cremanns, K.; Göttert, J. Photochemistry with Cyanines in the Near Infrared: A Step to Chemistry 4.0 Technologies. Chem. - A Eur. J. 2019, 25 (56), 12855-12864.

(17) Lim, K. S.; Galarraga, J. H.; Cui, X.; Lindberg, G. C. J.; Burdick, J. A.; Woodfield, T. B. F. Fundamentals and Applications of Photo-Cross-Linking in Bioprinting. Chem. Rev. 2020, acs.chemrev.9b00812.

(18) Schwartz, J. J.; Boydston, A. J. Multimaterial Actinic Spatial Control 3D and 4D Printing. Nat. Commun. 2019, 10 (1), 791.

(19) Bialas, S.; Michalek, L.; Marschner, D. E.; Krappitz, T.; Wegener, M.; Blinco, J.; Blasco, E.; Frisch, H.; Barner-Kowollik, C. Access to Disparate Soft Matter Materials by Curing with Two Colors of Light. Adv. Mater. 2019, 31 (8), 1807288.

(20) Dolinski, N. D.; Page, Z. A.; Callaway, E. B.; Eisenreich, F.; Garcia, R. V.; Chavez, R.; Bothman, D. P.; Hecht, S.; Zok, F. W.; Hawker, C. J. Solution Mask Liquid Lithography (SMaLL) for One-Step, Multimaterial 3D Printing. Adv. Mater. 2018, 30 (31), 1800364.

(21) Zhang, X.; Xi, W.; Huang, S.; Long, K.; Bowman, C. N. Wavelength-Selective Sequential Polymer Network Formation Controlled with a Two-Color Responsive Initiation System. Macromolecules 2017, 50 (15), 56525660 .

(22) Kottisch, V.; Michaudel, Q.; Fors, B. P. Photocontrolled Interconversion of Cationic and Radical Polymerizations. J. Am. Chem. Soc. 2017, 139 (31), 10665-10668.

(23) Peterson, B. M.; Kottisch, V.; Supej, M. J.; Fors, B. P. On Demand Switching of Polymerization Mechanism and Monomer Selectivity with Orthogonal Stimuli. ACS Cent. Sci. 2018, 4 (9), 1228-1234.

(24) Garra, P.; Dietlin, C.; Morlet-Savary, F.; Dumur, F.; Gigmes, D.; Fouassier, J. P.; Lalevée, J. Redox TwoComponent Initiated Free Radical and Cationic Polymerizations: Concepts, Reactions and Applications. Prog. Polym. Sci. 2019, 94, 33-56.

(25) Blasco, E.; Wegener, M.; Barner-Kowollik, C. Photochemically Driven Polymeric Network Formation: Synthesis and Applications. Adv. Mater. 2017, 29 (15), 1604005.

(26) Matsui, J. K.; Lang, S. B.; Heitz, D. R.; Molander, G. A. Photoredox-Mediated Routes to Radicals: The Value of Catalytic Radical Generation in Synthetic Methods Development. ACS Catal. 2017, 7 (4), 2563-2575.

(27) Dadashi-Silab, S.; Doran, S.; Yagci, Y. Photoinduced Electron Transfer Reactions for Macromolecular Syntheses. Chem. Rev. 2016, 116 (17), 10212-10275.

(28) Chen, M.; Zhong, M.; Johnson, J. A. Light-Controlled Radical Polymerization: Mechanisms, Methods, and Applications. Chem. Rev. 2016, 116 (17), 10167-10211.

(29) Haas, M.; Radebner, J.; Eibel, A.; Gescheidt, G.; Stueger, H. Recent Advances in Germanium-Based Photoinitiator Chemistry. Chem. - A Eur. J. 2018, 24 (33), 8258-8267.

(30) Bagheri, A.; Bainbridge, C. W. A.; Engel, K. E.; Qiao, G. G.; Xu, J.; Boyer, C.; Jin, J. Oxygen Tolerant PETRAFT Facilitated 3D Printing of Polymeric Materials under Visible LEDs. ACS Appl. Polym. Mater. 2020, 2 (2), 782-790.

(31) Zhang, Z.; Corrigan, N.; Bagheri, A.; Jin, J.; Boyer, C. A Versatile 3D and 4D Printing System through Photocontrolled RAFT Polymerization. Angew. Chemie - Int. Ed. 2019, 58 (50), 17954-17963. 
(32) Polykarpov, A. Y.; Neckers, D. C. Tetramethylammonium Phenyltrialkylborates in the Photoinduced Electron Transfer Reaction with Benzophenone. Generation of Alkyl Radicals and Their Addition to Activated Alkenes. Tetrahedron Lett. 1995, 36 (31), 5483-5486.

(33) Kabatc, J.; Jędrzejewska, B.; Pączkowski, J. Hemicyanine N-Butyltriphenylborate Salts as Effective Initiators of Free-Radical Polymerization Photoinitiated via Photoinduced Electron-Transfer Process. J. Polym. Sci. Part A Polym. Chem. 2003, 41 (19), 3017-3026.

(34) Pritchard, Z. D.; Beer, M. P.; Whelan, R. J.; Scott, T. F.; Burns, M. A. Modeling and Correcting Cure-Through in Continuous Stereolithographic 3D Printing. Adv. Mater. Technol. 2019, 1900700.

(35) Cui, G.; Guan, P. J.; Fang, W. H. Photoinduced Proton Transfer and Isomerization in a Hydrogen-Bonded Aromatic Azo Compound: A CASPT2//CASSCF Study. J. Phys. Chem. A 2014, 118 (26), 4732-4739.

(36) Shanmugam, S.; Xu, J.; Boyer, C. Exploiting Metalloporphyrins for Selective Living Radical Polymerization Tunable over Visible Wavelengths. J. Am. Chem. Soc. 2015, 137 (28), 9174-9185.

(37) Park, H. Y.; Kloxin, C. J.; Fordney, M. F.; Bowman, C. N. Stress Relaxation of TrithiocarbonateDimethacrylate-Based Dental Composites. Dent. Mater. 2012, 28 (8), 888-893.

(38) Ligon, S. C.; Husár, B.; Wutzel, H.; Holman, R.; Liska, R. Strategies to Reduce Oxygen Inhibition in Photoinduced Polymerization. Chem. Rev. 2014, 114 (1), 577-589.

(39) Monzón, M.; Ortega, Z.; Hernández, A.; Paz, R.; Ortega, F. Anisotropy of Photopolymer Parts Made by Digital Light Processing. Materials (Basel). 2017, 10 (1), 64.

(40) Dizon, J. R. C.; Espera, A. H.; Chen, Q.; Advincula, R. C. Mechanical Characterization of 3D-Printed Polymers. Addit. Manuf. 2018, 20, 44-67.

(41) Anastasio, R.; Peerbooms, W.; Cardinaels, R.; Van Breemen, L. C. A. Characterization of Ultraviolet-Cured Methacrylate Networks: From Photopolymerization to Ultimate Mechanical Properties. Macromolecules 2019, 52 (23), 9220-9231.

(42) Patel, D. K.; Sakhaei, A. H.; Layani, M.; Zhang, B.; Ge, Q.; Magdassi, S. Highly Stretchable and UV Curable Elastomers for Digital Light Processing Based 3D Printing. Adv. Mater. 2017, 29 (15).

(43) Herzberger, J.; Sirrine, J. M.; Williams, C. B.; Long, T. E. Polymer Design for 3D Printing Elastomers: Recent Advances in Structure, Properties, and Printing. Prog. Polym. Sci. 2019, 97.

(44) Thrasher, C. J.; Schwartz, J. J.; Boydston, A. J. Modular Elastomer Photoresins for Digital Light Processing Additive Manufacturing. ACS Appl. Mater. Interfaces 2017, 9 (45), 39708-39716. 